\title{
Fuzzy Cost Metric based Adaptive (FCMA) Routing Protocols for WSNs
}

\author{
Deepti Gupta* and Ajay K Sharma \\ Department of Computer Science and Engineering, \\ National Institute of Technology, Jalandhar, Punjab, India \\ *deepti_gupta49@yahoo.co.in and sharmaajayk@nitj.ac.in
}

\begin{abstract}
In this paper, the authors have proposed Fuzzy Cost Metric based Adaptive (FCMA) routing protocols for WSNs. Residual energy, Congestion level and Connectivity parameters have been used to calculate the fuzzy routing cost in order to decide the forwarding node. The results obtained indicate that the proposed FCMA protocols reduce the energy consumption and latency and increase the lifetime of the overall network.
\end{abstract}

Keywords: Fuzzy logic, cost metric, constrained flooding, real-time search, adaptive tree, wireless sensor networks

\section{Introduction}

Wireless sensor networks (WSNs) are composed of a large number of sensor devices that communicate with each other through wireless medium and have limited energy and computing capabilities [1]. It is usually the wireless communication component that consumes highest energy in a sensor network therefore, communication protocols are desired to be energy efficient. There are a wide number of applications where wireless sensor networks are being deployed. Few of these include industrial monitoring, building and home automation, medicine, environmental monitoring, urban sensor networks and energy management [2]. These networks are also used for security, military defence, disaster monitoring and prevention.

The wireless sensor networks are energy constrained due to the limited battery power of the sensor nodes. Therefore, the design of effective and efficient routing schemes that can support information exchange and processing in wireless sensor networks is a complex task. This further depends on the requirements of the application. Thus the motivation of our work is the design of a protocol for an energy-constrained wireless sensor network using fuzzy logic.

\section{Related work}

Many routing protocols for WSN have been proposed for wireless sensor networks. A detailed survey of these routing protocols for WSNs has been given in [3]. Energyefficient routes computed using the minimum energy path, maximum residual energy path, path with minimum number of hops to sink have been suggested in [4]. However, selecting the route with minimum energy or maximum residual energy can lead to depletion of the energy of sensor nodes decreasing the life of WSN as argued by Chang et al., [5]. Rahul et al., [6] have proposed an energy aware routing protocol that keeps a 
set of minimal energy paths and selects one of these sub-optimal paths randomly. This significantly increases the network lifetime.

Integer programming problem [5] and convex nonlinear optimization techniques [7] have been suggested to formulate the problem of maximizing network lifetime by energy aware routing. In [8] the authors have suggested an energy-aware multi-path routing approach in which traffic is spread over the nodes lying on different paths connecting the source to the sink, in proportion to their residual energy. In [9], an energy-aware routing algorithm has been proposed in which a cost function in terms of energy conservation, delay optimization and other performance metrics has been defined between two sensor nodes. The existing techniques focus on changing the routing metric in order to determine the best path between source and destination.

These approaches use fixed or crisp metrics and mathematical modelling for calculating energy-aware routing metrics. These metrics vary widely with the type of sensor node implementation platform [10] rendering these approaches inflexible and non adaptive to changes. In addition, factors used for calculating the cost metric for routing are conflicting. For instance, multiple hop communication reduces the transmission power but leads to greater number of hops thereby reducing the energy of relaying nodes.

Fuzzy logic [11], a decision system approach which works similarly to human control logic, however, is well suited for applications with such conflicting situations and imprecision in data using heuristic human reasoning without needing complex mathematical modelling. Moreover, fuzzy logic has the advantage of being easily adaptable to changes in energy metrics with change in sensor node platform. Furthermore, the execution requirements of fuzzy logic can be easily supported by sensor nodes. Thus better network performance is obtained. It allows combining and evaluating different parameters in an efficient manner. This technique uses human language to describe inputs and outputs. It provides a simplistic approach for obtaining a conclusion from imprecise and ambiguous information and provides quite accurate results. The system using fuzzy logic requires less computational power for execution than the conventional mathematical methods such as arithmetic operations [12]. Inference engine is used for performing calculations in a fuzzy logic based system. It consists of four phases: fuzzification, rule evaluation, combination or aggregation of rules, and deffuzification. The most commonly used inference engine is Mamdani [13].

In [14] a fuzzy logic based method has been implemented on ZigBee nodes. This method reduces the on/off frequency of an air conditioner system. The experimental results obtained are better than the ones from traditional control system based on discrete temperature values. Fuzzy logic has been used in [15] to perform link quality estimation. The results obtained from the experiments depict increase in reliability and stability of the network. In [16] the authors have proposed LEACH-FL based on fuzzy logic that takes node battery level, node density and distance to the base station as inputs for the selection of cluster-heads. This method leads to reduction in energy consumption and increase in network lifetime. The use of the output of a fuzzy logic based system in place of the metric of the Tree Routing protocol used in ZigBee in [17] has seen to result in reduction in the path length, the network discovery time and the number of forwarding nodes. Thus it has been observed that fuzzy logic can be used in wireless sensor network approaches, as it provides effective parameter combinations and is able to be executed in the low-resourced nodes that compose these networks.

In the constrained flooding routing protocol [18, 19] the authors have used the shortest path routing objective to forward data. In [20] the authors have suggested 
energy-aware and congestion-aware routing objectives. The evaluation of the constrained flooding (CF), real-time search (RTS) and adaptive tree (AT) routing protocols using energy-aware and congestion-aware routing objective have been studied in [21] and [22] respectively.

In this paper, we present a fuzzy model based adaptive scheme for calculating the cost of link between any two sensors nodes. The objective of our adaptive scheme is to determine the value of cost for a link between two sensor nodes such that the lifetime of the sensor network is maximized and the routing load is balanced effectively amongst the sensor nodes. The next section gives a detailed description of Fuzzy Cost Metric based Adaptive FCMA routing protocols for wireless sensor networks. The proposed routing scheme makes use of fuzzy logic to evaluate parameters of sensor nodes, the quality of links between them and the network conditions for creation of minimum cost route between any two nodes.

\section{Proposed Protocols}

The authors have defined a new cost metric named as Fuzzy cost based on the combination of node, network and link parameters using fuzzy logic. The input parameters are specified in natural language. A fuzzy rule set is used to define the relationship of the inputs with the output. This decides the suitability of a node to be selected as a part of the routing path. Figure 1 displays the fuzzy model used. The input fuzzy variables are: residual energy, congestion level and connectivity

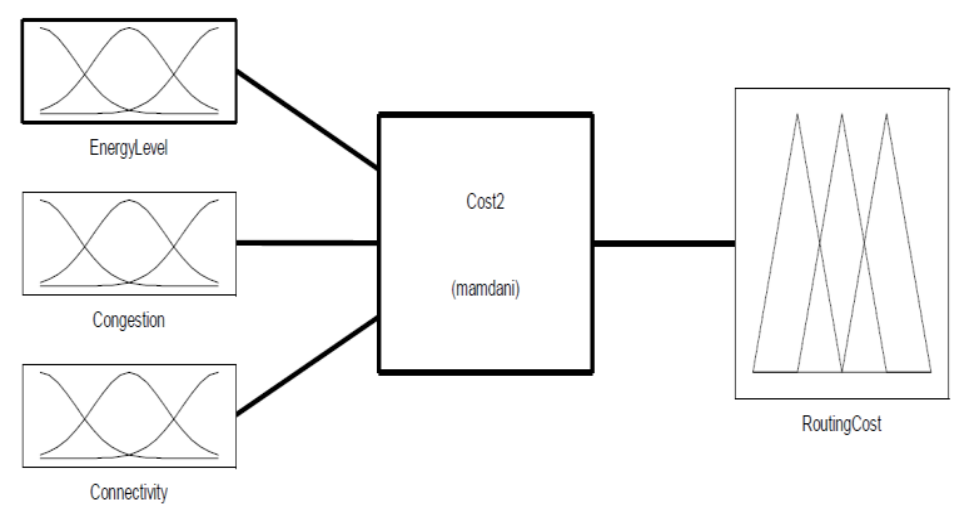

Figure 1. Fuzzy Cost Metric System Model

- Residual Energy: it indicates the current energy level of node $j$ when determining the cost of link from node $i$ to node $j$. This metric prevents nodes with less value of residual energy from taking part in routing paths and selected as next-hop. Consequently, its lower value results in a higher link cost. Route formation using nodes with high levels of residual energy will aid in saving the energy of battery powered nodes. This parameter helps in load balancing.

- Congestion level: it depicts the level of congestion at node $j$. Nodes with high congestion level are assigned higher link costs. This parameter ensures low latency and reliable communication. 
- Connectivity: it specifies the strength or power of signal between node $i$ and $j$. This parameter enables selection of routes with maximum signal strength and nodes with acceptable level of communication between them.

The rule base consists of 27 rules structured as follows:

IF Residual Energy is Low, AND Congestion level is High, AND Signal Strength is Medium THEN Fuzzy Cost is High.

The output fuzzy variable is named as Fuzzy cost. A cost between 0 and 100 is assigned to each link. The defuzzified value of the output variable determines the cost of link between two sensor nodes. MIN-MAX inference technique and centroid defuzzification method have been used in the fuzzy controller. In order to decide the node which will forward data, nodes compare Fuzzy cost obtained as a result of the evaluation of the input variables using fuzzy with their reachable neighbours. The node that has the lowest value of the Fuzzy cost among their reachable neighbours becomes the forwarding node. This method selects network paths comprising nodes with high residual energy, less congestion and better connectivity with their neighbors. This scheme allows high-resourced nodes to perform forwarding tasks, thus balancing load and discarding nodes that cannot provide acceptable signal strength.

\section{Results and Discussions}

We analyze the performance of the routing protocols using PROWLER (Probabilistic Wireless Network Simulator) and RMASE (Routing Modeling Application Simulation Environment). The normal radio model (NRM) in PROWLER attempts to simulate the probabilistic nature in wireless sensor communication observed by many [23]. We use a real application to test the performance of the Fuzzy cost metric. The application, Pursuer Evader Game (PEG) [24], uses the sensor network to detect an evader and to inform the pursuer about its location. In our tests, the network is a $7 \times 7$ sensor grid with small random offsets. The maximum radio range is about $3 d$, where $d$ is the standard distance between two neighbor nodes in the grid. The radio data rate is $40 \mathrm{kbps}$ [25] and each packet has 960 bits. The application sends out one packet per second from the sources. The results are based on the average of 10 random runs.

This section discusses the simulation results of the comparative investigation of the performance of the proposed Fuzzy Cost Metric based Adaptive routing protocols for wireless sensor network.

\subsection{Case 1: FCMA-CF}

Figure 2 shows that the energy consumed by FCMA-CF is considerably lower than that consumed by CF. The energy consumed per round is 0.37 and 0.04 in case of CF and FCMA$\mathrm{CF}$ respectively. It can be observed that there is $95 \%$ decrease in energy consumption in case of FCMA-CF. This energy saving is due to the reduction in the number of packets sent during routing. The number of packets sent and received directly affects energy consumption. This reduction allows WSNs running FCMA-CF to increase their lifetime. 


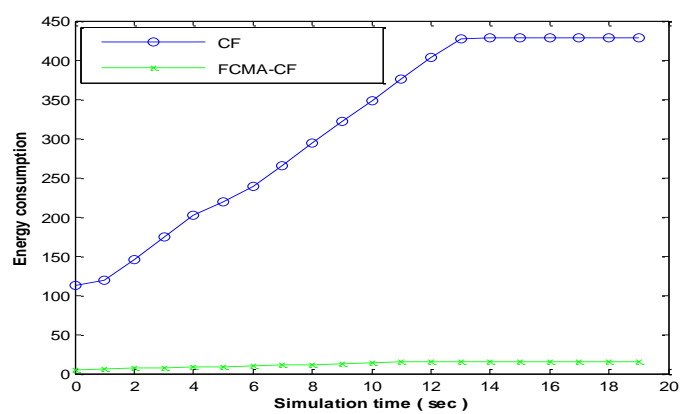

Figure 2. Energy consumption of CF vs. FCMA-CF

Figure 3 indicates that the lifetime of sensor networks in case of $\mathrm{CF}$ varies between (1996.5-1988) years while it lies in the range of (1999.5-1998.5) years for FCMA-CF. An increase of 10.5 years in network lifetime at simulation time $13 \mathrm{sec}$ is observed. This is because when Fuzzy cost metric is used to update the routing cost in the network there is decrease in energy consumption by the sensor nodes.

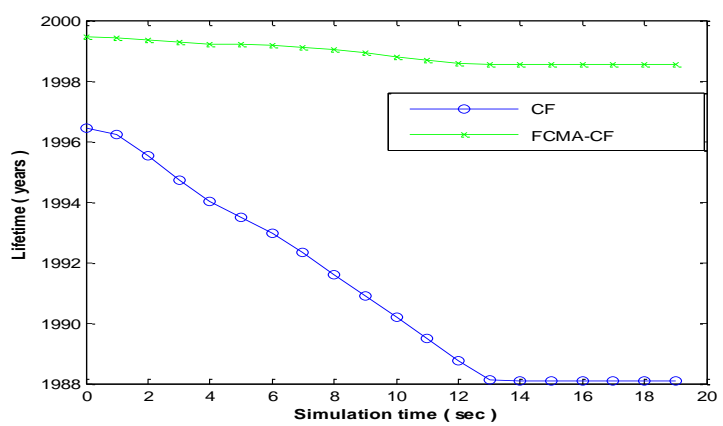

Figure 3. Lifetime of CF vs. FCMA-CF

Figure 4 depicts the latency for CF and FCMA-CF. It is observed that the latency is 0.13 and 0.02 seconds respectively in case of CF and FCMA-CF at the end of simulation time of $13 \mathrm{sec}$. Thus it can be concluded that the network latency is reduced by $85 \%$ when FCMA-CF is used in the sensor network. This is due to the reduced number of collisions of the packets reaching the destination nodes running FCMA-CF.

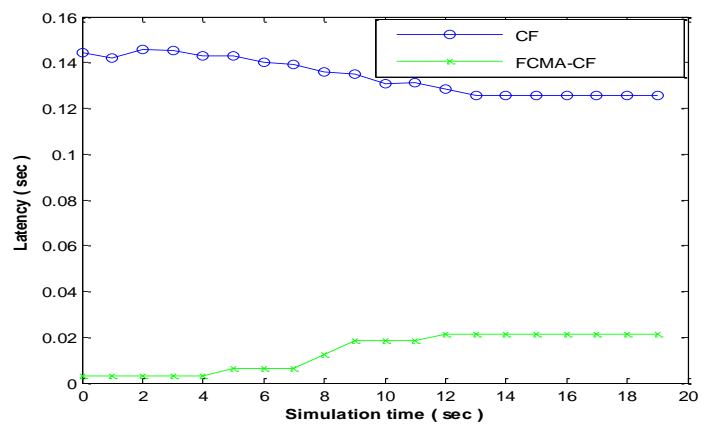

Figure 4. Latency of CF vs. FCMA-CF 


\subsection{Case 2: FCMA-RTS}

Figure 5 shows that the energy consumed by FCMA-RTS is much lower than that consumed by RTS. The energy consumed per round is 0.05 and 0.02 in case of RTS and FCMA-RTS respectively. Thus $75 \%$ energy saving is achieved for WSNs running FCMARTS.

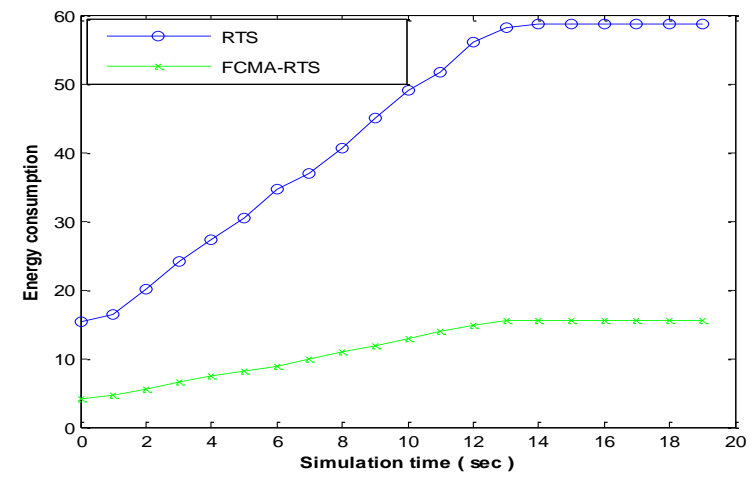

Figure 5. Energy consumption of RTS vS. FCMA-RTS

Figure 6 indicates that the lifetime of sensor networks for RTS and FCMA-RTS varies in the range (1999-1996) and (1999.5-1998.5) years respectively. Thus there is an increase of 2.5 years at the end of simulation time of $13 \mathrm{sec}$ when FCMA-RTS is used in WSNs. This is the result of less energy consumption by the nodes in the network.

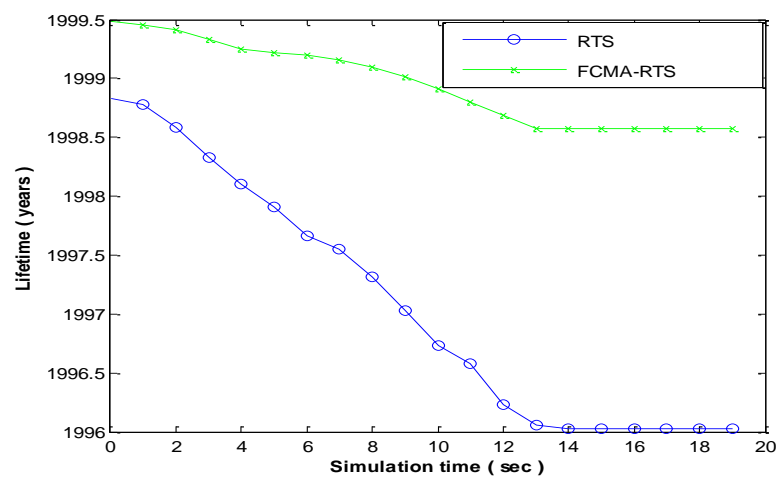

Figure 6. Lifetime of RTS vs. FCMA-RTS

Figure 7 depicts the latency for RTS and FCMA-RTS. The latency is 0.03 seconds in case of FCMA-RTS and 0.1 seconds in case of RTS at the end of simulation time of 13 seconds. Thus it can be concluded that the network latency is reduced by $70 \%$ when FCMA-RTS is used in the sensor network. 


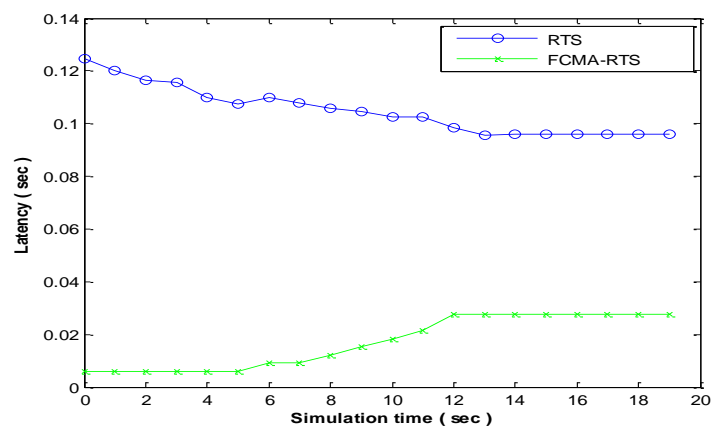

Figure 7. Latency of RTS vs. FCMA-RTS

\subsection{Case 3: FCMA-AT}

Figure 8 shows that the energy consumed by FCMA-AT is less than that consumed by AT. The energy consumed per round is 0.06 and 0.02 in case of AT and FCMA-AT respectively. Thus FCMA-AT consumes $80 \%$ less energy as compared to AT.

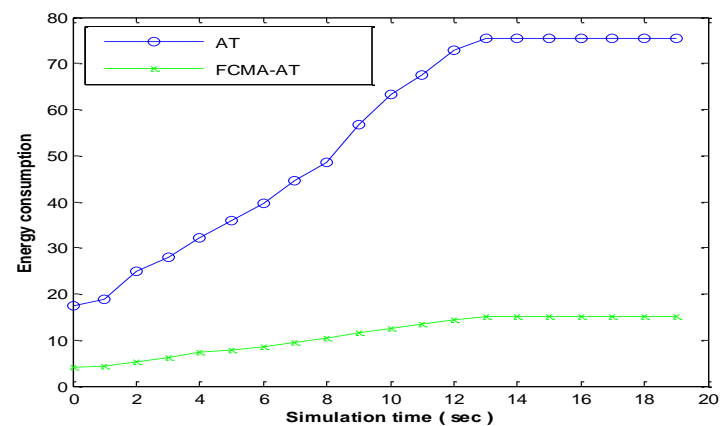

Figure 8. Energy consumption of AT vs. FCMA-AT

Figure 9 indicates that the lifetime of sensor networks falls between (1998.5-1994.5) and (1999.5-1998.5) years respectively in case of AT and FCMA-AT. An increase in the network lifetime by 4 years for FCMA-AT using Fuzzy cost metric to update the routing cost in the network. This is in turn is the result of decreased energy consumption by the sensor nodes running FCMA-AT.

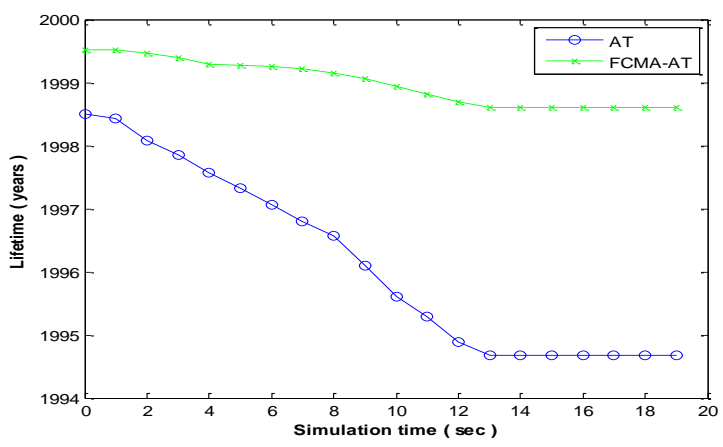

Figure 9. Lifetime of AT vs. FCMA-AT 
Figure 10 depicts the latency for AT and FCMA-AT. The latency is 0.09 seconds and 0.03 seconds respectively for AT and FCMA-AT. Thus it can be concluded that the network latency is decreased by $67 \%$ when FCMA-AT is used in the sensor network. Thus the use of the Fuzzy cost metric for updating the routing cost for the node reduces the latency with which packets arrive at the destination.

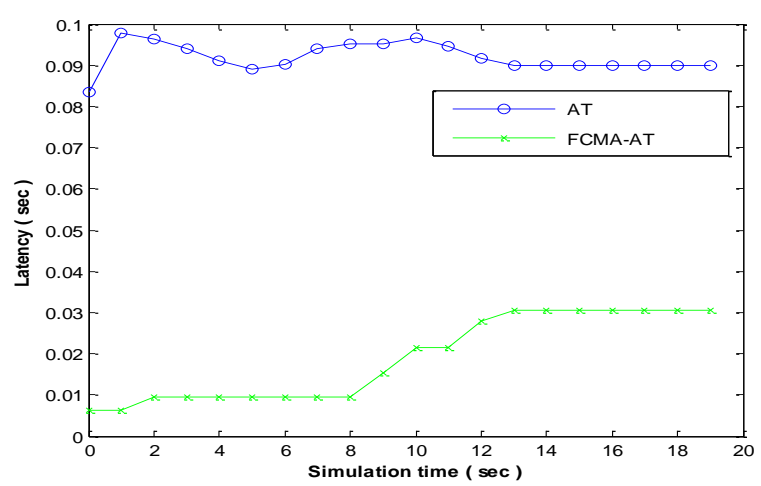

Figure 10. Latency of AT vs. FCMA-AT

It has been concluded that there is a decrease in energy consumption by $95 \%, 75 \%$ and $80 \%$ in case FCMA-CF, FCMA-RTS and FCMA-AT respectively. Consequently, the network lifetime is increased by 10.5 years, 2.5 years, and 4 years for FCMA-CF, FCMARTS and FCMA-AT respectively. Moreover, the latency of the network is reduced by $85 \%$, $70 \%$, and $67 \%$ in case of FCMA-CF, FCMA-RTS and FCMA-AT respectively.

\section{Conclusions}

This work proposes a fuzzy-logic-based approach for calculating the routing cost that improves the performance of the constrained flooding, real-time search and adaptive tree routing protocols. The use of residual energy, congestion, and signal strength and their evaluation by using a fuzzy logic approach has been proposed for determining the routing cost. From results obtained we can observe that FCMA-CF, FCMA-RTS and FCMA-AT (Fuzzy Cost Metric based Adaptive CF, RTS and AT) consume less energy, since it sends less messages resulting in fewer collisions and the latency is also reduced. Therefore, the use of fuzzy logic as a metric in network routing improves the performance of the overall network. It has been concluded that the proposed fuzzy-logic-based decision making scheme improves network performance, in terms of energy consumption, lifetime, energy efficiency, latency. Moreover, the incorporation of this mechanism does not leads to an extra energy consumption, making this scheme appropriate to be used in routing techniques for wireless sensor networks. The comparative performance investigations with CF, RTS and AT suggest the suitability of FCMA-CF, FCMA-RTS and FCMA-AT for implementation in real wireless sensor networks. Further, the fuzzy logic is an excellent tool to be used in WSN approaches, since it provides effective parameter combination, and it is able to be executed in the lowresourced nodes comprising these networks. The input variables and rules used are highly application specific. These can be customized depending on the application requirements, node features and network size. 


\section{References}

[1] J. Yick, B. Mukherjee and D. Ghosal, "Wireless Sensor Network Survey", Computer Networks, vol. 52, (2008).

[2] J. P. Vasseur, "Terminology in Low power And Lossy Networks", Internet draft, Networking Working Group, 2010. http://tools.ietf.org/html/draft-ietf-roll-terminology-04.

[3] K. Akkaya and M. Younis, "A Survey of Routing Protocols in Wireless Sensor Networks", Computer Journal of Elsevier Ad Hoc Network Journal, vol. 3, no. 3, (2005), pp. 325-349.

[4] F. Akyildiz, "A Survey on Sensor Networks", Computer Journal of IEEE Communications Magazine, vol. 40, no. 8, (2002), pp. 102-114.

[5] H. Chang, S. Leandros and L. Tassiulas, "Maximum Lifetime Routing in Wireless Sensor Networks", Computer Journal of IEEE/ACM Transactions on Networking, vol. 12, no. 4, (2004), pp. 609-619.

[6] R. Shah and J. Rabaey, "Energy Aware Routing for Low Energy Ad Hoc Sensor Networks", Proceedings of the IEEE Wireless Communications and Networking Conference (WCNC2002), Orland, (2002), pp. 350-355.

[7] B. Krishnamachari and F. Ord'o, "Analysis of Energy Efficient Fair Routing in Wireless Sensor Networks Through non Linear Optimization", Proceedings of Workshop on Wireless Ad Hoc Sensor and Wearable Networks, IEEE Vehicular Technology Conference, Florida, (2003), pp. 2844-2848.

[8] N. Jain, D. Madathil and D. Agrawal, "Energy Aware Multi Path Routing for Uniform Resource Utilization in Sensor Networks", Proceedings of International Workshop on Information Processing in Sensor Networks (IPSN'03), California, (2003), pp. 392-404.

[9] M. Younis, M. Youssef and K. Arisha, "Energy: Aware Management for Cluster Based Sensor A Fuzzy Approach to Energy Optimized Routing for Wireless Sensor Networks 185 Networks", Computer Journal of Networks, vol. 43, no. 5, (2003), pp. 649-668.

[10] V. Raghunathan, C. Schurgers, S. Park and M. Srivastava, "Energy Aware Wireless Sensor Networks", Proceedings of IEEE Signal Processing, USA, (2002), pp. 174-188.

[11] L. Reznik, "Fuzzy Controllers", Newnes Publishing, Oxford, UK, (1997).

[12] W. Su and T. C. Bougiouklis, "Data fusion algorithms in clusterbased wireless sensor networks using fuzzy logic theory", 11th WSEAS International Conference on Communications, (2007).

[13] J. -S. R. Jang, C. T. Sun and E. Mizutani, "Neuro-fuzzy and soft computing: a computational approach to learn and machine intelligence", Prentice Hall, (1997).

[14] T. M. Wang, I. J. Liao, J. C. Liao, T. -W. Suen and W. T. Lee, “An Intelligent Fuzzy Controller for AirCondition with ZigBee Sensors", International Journal on Smart Sensing and Intelligent Systems, vol. 2, no. 4, (2009) December.

[15] N. Bacour, A. Koubaa, H. Youssef, M. B. Jamaa, D. do Rosario, M. Alves and L. B. Becker, "F-LQE: A Fuzzy Link Quality Estimator for Wireless Sensor Networks", Proceedings of the European Conference on Wireless Sensor Networks (EWSN), (2010).

[16] G. Ran, H. Zhang and S. Gong, "Improving on LEACH Protocol of Wireless Sensor Networks Using FuzzyLogic", Journal of Information and Computational Science, vol. 7, no. 3, (2010).

[17] A. M. Ortiz, T. Olivares and L. Orozco-Barbosa, "Smart Routing Mechanism for Green ZigBee-based Wireless Sensor Networks", Proceedings of the 16th IEEE Symposium on Computer and Communications (ISCC), (2011).

[18] Y. Zhang and M. Fromherz, "A robust and efficient flooding-based routing for wireless sensor networks", Journal of Interconnection Networks, vol. 7, no. 4, (2006), pp. 549-568.

[19] Y. Zhang and M. Fromherz, "Constrained Flooding: A robust and efficient routing framework for wireless sensor networks", 20th International Conference on Advanced Information Networking and Applications, (2006).

[20] Y. Zhang and Q. Huang, "A learning-based adaptive routing tree for wireless sensor networks", Journal of Communications, vol. 1, no. 2, (2006).

[21] D. Gupta and A. K. Sharma, "Performance Evaluation of Routing Protocols for WSNs Based on Energyaware Routing with different Radio Models", International Journal of Computer Applications (IJCA), vol. 3, no. 12, Article 2, (2010) July, pp. 6-14.

[22] D. Gupta and A. K. Sharma, "Performance Investigations of Routing Protocols for WSNs based on Congestion-Aware Routing with Different Radio Models", International Journal of Applied Engineering Research (IJAER), vol. 6, no. 12, (2011) September, pp. 1461-1480.

[23] G. Simon, "Prowler: Probabilistic Wireless Network Simulator", Institute for Software Integrated Systems (ISIS)", (2003), http://www.isis.vanderbilt.edu/projects/nest/prowler.

[24] Y. Zhang and M. Fromherz, "A robust and efficient flooding-based routing for wireless sensor networks", Journal of Interconnection Networks, vol. 7, no. 4, (2006), pp. 549-568.

[25] Crossbow: MICA, Wireless Measurement System Datasheet: http://www.xbow.com/Products/Product_pdf_files/Wireless_pdf/MICA.pdf. 


\section{Authors}

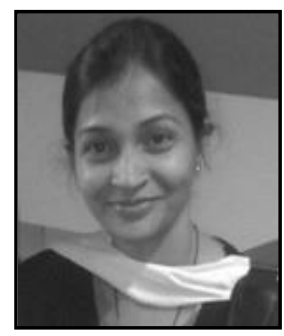

Deepti Gupta received her BE in Computer Science and Engineering from University of Jammu, Jammu and Kashmir, India in 2006 and MTECH in Computer Science and Engineering from Dr. B R Ambedkar National Institute of Technology, Jalandhar, Punjab, India in the year 2009. Her MTECH thesis was on "Performance Evaluation of Routing Protocols for Wireless Sensor Networks with Different Radio Models". She is currently pursuing full-time $\mathrm{PhD}$ in the Department of Computer Science and Engineering, Dr. B R Ambedkar National Institute of Technology, Jalandhar, Punjab, India. Her professional research activity lies in the field of wireless sensor networks.

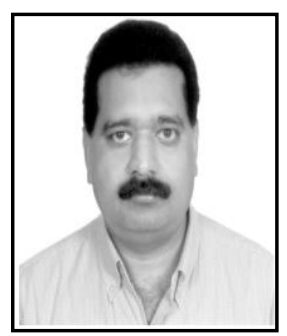

Ajay K Sharma received his BE in Electronics and Electrical Communication Engineering from Punjab University Chandigarh, India in 1986, MS in Electronics and Control from Birla Institute of Technology (BITS), Pilani in the year 1994 and $\mathrm{PhD}$ in Electronics Communication and Computer Engineering in the year 1999. His $\mathrm{PhD}$ thesis was on "Studies on Broadband Optical Communication Systems and Networks". From 1986 to 1995 he worked with TTTI, DTE Chandigarh, Indian Railways New Delhi, SLIET Longowal and National Institute of technology (Erstwhile Regional Engineering College), Hamirpur HP at various academic and administrative positions. He has joined National Institute of Technology (Erstwhile Regional Engineering College) Jalandhar as Assistant Professor in the Department of Electronics and Communication Engineering in the year 1996. From November 2001, he has worked as Professor in the ECE department and presently working as Professor in Computer Science \& Engineering in the same institute. His major areas of interest are broadband optical wireless communication systems and networks, dispersion compensation, fiber nonlinearities, optical soliton transmission, WDM systems and networks, Radio-over-Fiber (RoF) and wireless sensor networks and computer communication. He has published 262 research papers in the International/National Journals/Conferences and 12 books. $\mathrm{He}$ has supervised $15 \mathrm{Ph} . \mathrm{D}$. and $43 \mathrm{M}$.Tech theses. He has completed three R\&D projects funded by Government of India and one project is ongoing. Presently he was associated to implement the World Bank project of 209 Million for Technical Education Quality Improvement programme of the institute. He is technical reviewer of reputed international journals like: Optical Engineering, Optics letters, Optics Communication, Digital Signal Processing. He has been appointed as member of technical Committee on Telecom under International Association of Science and Technology Development (IASTD) Canada for the term 2004-2007 and he is Life Member of Optical Society of America, USA, (LM ID-361253), Computer Society of India, Mumbai, India, (LM-Associate: 01099298), Advanced Computing \& Communications Society, Indian Institute of Science, Bangalore, India, (L284M1100306), SPIE, USA, (ID: 619838), Indian Society for Technical Education (I.S.T.E.), New Delhi, India, (LM-11724), Fellow The Institution of Electronics and Telecommunication Engineers (IETE), (F-224647) . 